BMJ Open

Diabetes

Research

\& Care

\title{
Systemic AAV10.COMP-Ang1 rescues renal glomeruli and pancreatic islets in type 2 diabetic mice
}

\author{
Mi Tian, ${ }^{1,2}$ Lara S Carroll, ${ }^{3}$ Li Tang, ${ }^{1}$ Hironori Uehara, ${ }^{3}$ Christof Westenfelder, ${ }^{1}$ \\ Balamurali K Ambati, ${ }^{3}$ Yufeng Huang (D) ${ }^{1}$
}

To cite: Tian M, Carroll LS, Tang L, et al. Systemic AAV10. COMP-Ang1 rescues renal glomeruli and pancreatic islets in type 2 diabetic mice. BMJ Open Diab Res Care 2020;8:e000882. doi:10.1136/ bmjdrc-2019-000882

- Additional material is published online only. To view please visit the journal online (http://dx.doi.org/10.1136/ bmjdrc-2019-000882).

Received 5 September 2019 Revised 5 February 2020 Accepted 14 June 2020

\section{Check for updates}

(C) Author(s) (or their employer(s)) 2020. Re-use permitted under CC BY-NC. No commercial re-use. See rights and permissions. Published by BMJ.

${ }^{1}$ Internal Medicine/Nephrology, University of Utah, Salt Lake City, Utah, USA

${ }^{2}$ Internal Medicine/Nephrology, Shengjing Hospital of China Medical University, Shenyang, China

${ }^{3}$ Ophthalmology and Visual Science, John A. Moran Eye Center, University of Utah, Salt Lake City, Utah, USA

Correspondence to Dr Yufeng Huang; yufeng.huang@hsc.utah.edu

\section{ABSTRACT}

Introduction Diabetic hyperglycemia causes progressive and generalized damage to the microvasculature. In renal glomeruli, this results in the loss of podocytes with consequent loss of constitutive angiopoietin-1 (Ang1) signaling, which is required for stability of the glomerular endothelium. Repeated tail vein injection of adenovirus expressing COMP-Ang1 (a stable bioengineered form of Ang1) was previously reported to improve diabetic glomerular damage despite the liver and lungs being primary targets of adenoviral infection. We thus hypothesized that localizing delivery of sustained COMPAng1 to the kidney could increase its therapeutic efficacy and safety for the treatment of diabetes.

Research design and methods Using AAVrh10 adenoassociated viral capsid with enhanced kidney tropism, we treated 10-week-old uninephrectomized db/db mice (a model of type 2 diabetes) with a single dose of AAVrh10. COMP-Ang1 delivered via the intracarotid artery, compared with untreated diabetic $\mathrm{db} / \mathrm{db}$ control and non-diabetic $\mathrm{db} / \mathrm{m}$ mice.

Results Surprisingly, both glomerular and pancreatic capillaries expressed COMP-Ang1, compensating for diabetes-induced loss of tissue Ang1. Importantly, treatment with AAVrh10.COMP-Ang1 yielded a significant reduction of glycemia (blood glucose, $241 \pm 193 \mathrm{mg} / \mathrm{dL}$ vs $576 \pm 31 \mathrm{mg} / \mathrm{dL}$; glycosylated hemoglobin, $7.2 \pm 1.5 \%$ vs $11.3 \pm 1.3 \%$ ) and slowed the progression of albuminuria and glomerulosclerosis in $\mathrm{db} / \mathrm{db}$ mice by $70 \%$ and $61 \%$, respectively, compared with untreated diabetic $\mathrm{db} / \mathrm{db}$ mice. Furthermore, COMP-Ang1 ameliorated diabetes-induced increases of NF-kBp65, nicotinamide adenine dinucleotide phosphate (NAPDH) oxidase-2 (Nox2), p47phox and productions of myeloperoxidase, the inflammatory markers in both renal and pancreatic tissues, and improved betacell density in pancreatic islets.

Conclusions These results highlight the potential of localized Ang1 therapy for treatment of diabetic visceropathies and provide a mechanistic explanation for reported improvements in glucose control via Ang1/Tie2 signaling in the pancreas.

\section{INTRODUCTION}

Diabetes is the leading cause of end-stage renal disease (ESRD) and almost half of new patients with ESRD have diabetes. ${ }^{1}$ Diabetic nephropathy (DN) reduces the quality of life of diabetic individuals and is associated

\section{Significance of this study}

What is already known about this subject?

- Hyperglycemia causes progressive and generalised damage to the microvasculature. However, there are no clinical antivasodegenerative treatments for diabetes and diabetes-related complications.

What are the new findings?

- The current study designed a novel long-term expression of the AAVrh10.COMP-Ang1 plasmid, which results in sustained gene expression of COMP-Ang1 with a signal arterial injection directed to renal glomerular and pancreatic islet capillaries in type $2 \mathrm{di}-$ abetic $\mathrm{db} / \mathrm{db}$ mice.

- Localized COMP-Ang1 expression not only improves pancreatic function and hyperglycemia but also slows the progression of diabetes-associated glomerulosclerosis by improving both pancreatic and glomerular endothelial functions via activating the Ang1-Tie2 signaling.

How might these results change the focus of research or clinical practice?

- Our study suggests that AAVrh10.COMP-Ang1 can be a future focus, as a promising, capillary endothelium-targeted gene therapeutic for type 2 diabetes.

with an overall increased cardiovascular risk resulting in increased mortality and morbidity. ${ }^{2}$ However, the underlying pathogenic mechanisms have yet to be completely elucidated. Microvascular dysfunction, specifically injury to the capillary endothelium, has been suggested to be a critical component of DN pathogenesis similar to that observed in diabetic retinopathy. Currently, there is no clinical antivasodegenerative treatment for DN. Additional therapies for DN that target vascular dysfunction remain a clear unmet medical need.

Increasing evidence indicates that glomerular endothelial damage is already present in the normoalbuminuric stage of diabetes in both type 1 and type 2 diabetes. This damage 
includes abnormal new vessel generation with immature endothelial cells, a thin wall, and reduced negative charge components, which contributes to glomerular hypertrophy and increased protein trafficking through the endothelium and thereby subsequently causing or enhancing podocyte injury. ${ }^{3-5}$ Such early abnormal change of glomerular endothelium in diabetic kidneys is likely linked to progressive ischemia and inflammation associated with later stages of the disease. Treatments designed to target glomerular endothelium in the early phase of DN may save the kidney.

It has been shown that angiopoietins and their receptor play a critical role for microvascular endothelium hemostasis. The angiopoietins (Ang1 and Ang2) are secreted ligands that bind to the receptor tyrosine kinase Tie2 expressed on many cell types, including vascular endothelium and hematopoietic cells. Ang1 binding to Tie2 on endothelium induces vessel survival, quiescence and maturation, and decreases inflammation and leakiness in response to permeability-inducing inflammatory agents. ${ }^{6}$ In contrast, Ang2 can compete with and antagonize Ang1 binding to the Tie2 receptor, hence reducing Tie2 activation and signaling on endothelium, particularly in diabetes and other microangiopathic conditions. ${ }^{7}$ Within the healthy glomerular capillary, Ang1 is mainly expressed in podocytes, while Ang2 is undetectable, being normally expressed only during glomerular development and by immature mesangial cells. The Tie2 receptor is expressed primarily on endothelial cells and podocytes. ${ }^{8}$ In both humans and animals with both type 1 and type 2 diabetes, Ang2 is found to be upregulated, ${ }^{9}{ }^{10}$ resulting in excess expression of Ang2 over Ang1 and secondary Tie2 receptor dysregulation. Overexpression of Ang2 in a mouse model with inducible podocyte-specific Ang2 expression resulted in glomerular endothelial apoptosis and albuminuria. ${ }^{11}$ Genetic ablation of Ang1 in podocytes led to further disruption of the diabetes-induced local imbalance of Ang1 and Ang2 and resulted in more severe glomerular endothelial lesions and proteinuria in diabetes. ${ }^{12}$ In contrast, podocyte-specific inducible repletion of Ang1 in diabetic mice caused a $70 \%$ reduction of albuminuria and prevented diabetes-induced glomerular endothelial cell proliferation. ${ }^{13}$ Furthermore, a recent study showed that addition of Ang1 in isolated glomeruli reduced vascular permeability by modifying the endothelial cell glycocalyx layer. ${ }^{14}$ Collectively, these observations suggest that podocyte secretion of Ang1 is required for endothelial cell maturation and stability. Importantly, Ang2 levels either in patients' serum, urine, or in renal tissue were significantly elevated in diabetes compared with healthy subjects and these levels are even higher in diabetic patients with microalbuminuria than those without albuminuria, indicating that a decreased secretion of Ang1 or ratio of Ang1 to Ang2 (or a reduced Ang1-Tie2 signaling) in the kidney may play a key role in the pathophysiology of $\mathrm{DN}$ in diabetes. Thus, reinstating Tie2 signaling by enhancing Ang1 production and modulating the glomerular balance between Ang1 and Ang2 is a promising therapeutic target in DN.

Similar to renal glomeruli, the pancreatic islet is highly vascularized with a high-density and high-permeability microcirculation. Within the pancreatic islet, the role of Ang1-Tie2 signaling in diabetes remains largely unknown. However, improvement in islet vascularization and function of newly implanted islets was achieved in diabetic mice by enhancing islet Ang1 production with an expression plasmid ${ }^{15}$ or by inhibition of Ang2 with a specific inhibitor. ${ }^{16}$ In either case, vascular or beta-cell density of islet grafts was improved concurrently with metabolic function of the diabetic recipients. These data highlight an important role of Tie2 signaling in pancreatic vascular defects that may be impaired by hyperglycemia in diabetes.

We thus proposed to use a well-established experimental diabetes model to investigate whether replacing constitutive Ang1 (lost from supporting cells due to hyperglycemia) with a single arterial injection of a novel adenoassociated virus, AAVrh10.COMP-Ang1, could effectively target visceral organs to protect the remaining endothelial cells in both pancreas and renal glomeruli, thereby ameliorating beta-cell function and glomerulosclerosis.

\section{RESEARCH DESIGN AND METHODS}

\section{Animals}

Diabetic male $\mathrm{db} / \mathrm{db}$ mice and their lean non-diabetic $\mathrm{db} / \mathrm{m}$ littermates were obtained from the Jackson Laboratory (Bar Harbor, Maine, USA). The maintenance and all study procedures of animals described herein were performed according to the guidelines of the Public Health Service Policy on Use of Laboratory Animals and were approved by the Animal Care Committee of University of Utah.

The $\mathrm{db} / \mathrm{db}$ mice were determined to be diabetic by the vendor on the basis of appearance of obesity at the age of approximately week 5 , and were further verified by multiple measurements of blood glucose (BG) and glycosylated hemoglobin (HbAlc) levels in our laboratory beginning at week 7 . All $\mathrm{db} / \mathrm{db}$ mice listed in the following studies were subjected to right uninephrectomy under anesthesia at week 8 to hasten the development of $\mathrm{DN}$ as described previously. ${ }^{17}$ Age-matched uninephrectomized $\mathrm{db} / \mathrm{m}$ mice served as experimental controls.

\section{Virus vector construction}

The recombinant AAV plasmid encoding COMP-Ang1 (pAAV.COMP-Ang1) was descried previously. ${ }^{18} 19$ The pcDNA vector contains a secretory signal sequence for hemagglutinin and a FLAG tag. This vector was used to express the coiled-coil domain of rat cartilage oligomeric matrix protein (COMP) fused to the fibrinogenlike domain of angiopoietin-1 (Ang1). It has been shown that the C-terminal fibrinogen-like domain of Ang1 is responsible for Tie2 receptor binding. ${ }^{20}$ The minimal coiled coil of COMP allows for the Ang1 to oligomerize 
without aggregating, which is necessary for its activity and stability, respectively. ${ }^{18}$ The therapeutic inhibitory function of the soluble recombinant COMP-Ang1 protein has been confirmed on in vivo VEGF-induced cornea angiogenesis. ${ }^{18}$ Previous success with intravenous delivery of adenovirus expressing COMP-Ang1 (Ade-COMP-Ang1) has been reported in the $\mathrm{db} / \mathrm{db}$ mouse model. ${ }^{21}$

However, limited expression duration required repeated injections. We recently reported long-term expression of AAV2.COMP-Ang1 in the retinas of diabetic Ins2akita mice for at least 6 months after a single intravitreal injection of AAV2.COMP-Ang1. ${ }^{19}$ Since the AAVrh10 serotype is reported to have increased tropism for the kidney with prolonged expression duration, ${ }^{22}$ we acquired AAVrh10.COMP-Ang1 (manufactured for us at the Vector Core Gene Therapy Center, University of Massachusetts Medical School). A pAAV.AcGFP plasmid containing the green fluorescent protein (GFP) coding sequence of pIRES2-AcGFP1 (Clontech Laboratories, Mountain View, California, USA ${ }^{19}$ was similarly packaged into AAVrh10.GFP to provide in vivo delivery controls.

\section{Experimental design \\ Study 1: determination of optimal route for delivery of AAVrh10. COMP-Ang1 to the kidney}

We first treated non-diabetic C57BL/6 mice with AAVrh10.GFP $\left(2 \times 10^{11}\right.$ particles $)$ for 2 weeks to track the GFP expression following tail vein injection (intravenous), left carotid artery injection or direct renal delivery with individual mini-pumps $(\mathrm{n}=2$ for each delivery method). (The detail procedures for artery injection and direct renal delivery are described in the online supplementary file.) The dose of AAVrh10.GFP was determined based on our previous study. ${ }^{19}$ After systemic perfusion with cold phosphate buffered saline (PBS), mouse organs were harvested for immunofluorescent detection of GFP protein.

\section{Study 2: effect of AAVrh10.COMP-Ang1 in diabetic db/db mice}

Groups of five to six uninephrectomized mice were assigned and treated as described, including (1) untreated non-diabetic $\mathrm{db} / \mathrm{m}$ mice $(\mathrm{n}=6)$ as healthy controls, (2) untreated diabetic $\mathrm{db} / \mathrm{db}$ mice $(\mathrm{n}=6)$ as a disease control, and (3) diabetic $\mathrm{db} / \mathrm{db}$ mice treated with a single dose of AAVrh10.COMP-Ang1 $\left(2 \times 10^{11}\right.$ particles) injected via the left carotid artery at 10 weeks of age $(n=5)$. The untreated $\mathrm{db} / \mathrm{m}$ and $\mathrm{db} / \mathrm{db}$ controls received PBS injection via the left carotid artery as the injection control. Mice were followed up to 8 weeks after treatment and were sacrificed at 18 weeks of age.

The BG level and HbA1c level were monitored in tail blood samples, and urinary albumin and the albumin/ creatinin $(\mathrm{Cr})$ levels were measured in 24-hour urine samples as described previously. ${ }^{17}$ Urine monocyte chemoattractant protein-1 (MCP-1) levels were measured using the commercially available ELISA kits (R\&D Systems, Minneapolis, Minnesota, USA). Urine malondialdehyde (MDA), also named as thiobarbituric acid-reactive substances (TBARS), which is derived from lipid hydroperoxides produced by oxidative stress and reacts with thiobarbituric acid was measured using a colorimetric assay (Cayman Chemical Company, Ann Arbor, Michigan, USA). The levels of MCP-1 or TBARS excretion were corrected by urine Cr levels.

Mice were euthanized under isoflurane anesthesia. Blood samples were obtained by cardiac puncture for the measurements of plasma blood urea nitrogen (BUN) and Cr by using the QuantiChrom urea and $\mathrm{Cr}$ assay kits (BioAssay System, Hayward, California, USA) and insulin levels by using the commercially available ELISA kits (Crystal Chem, ELK Grove Village, Illinois, USA). Kidneys were perfused through the heart with cold PBS and then excised. Renal cortex was harvested by dissection and saved for further analysis as described previously. ${ }^{17}$ Briefly, two pieces of renal cortex were either snap-frozen in 2-methylbutane at $-80^{\circ} \mathrm{C}$ or fixed in $10 \%$ neutralized formalin for immunohistochemistry staining. Other pieces of cortex were stored in liquid nitrogen for western blot or treated with Tri Reagent for isolation of RNA. In addition, the heart, liver, lung, pancreas and muscle were harvested for the determination of COMP-Ang1 expression. Immunohistochemical staining of inflammation and oxidative stress markers was further performed in pancreatic tissue.

\section{Histological analyses}

Formalin-fixed renal cortex tissues were subsequently embedded in paraffin. Three-micrometer sections were cut from the tissue blocks and stained with periodic acid-Schiff (PAS). The PAS-positive glomerular matrix was quantitated in a blinded fashion by a computerassisted method as previously described. ${ }^{17}$ At least 20 glomeruli from each individual mouse were assessed. The PAS-positive material area in the mesangium was normalized by that of the total glomerular tuft, where the percentage of mesangial matrix occupying each glomerulus area was rated. The average glomerular sclerosis was obtained by averaging scores from all glomeruli on one section. All examinations were performed independently in a blinded manner by two individuals.

Immunofluorescent staining for GFP and COMP-Ang1 was performed on frozen sections of kidney tissue and pancreatic tissue. The polyclonal chicken anti-GFP antibody (Invitrogen, Thermo Fisher Scientific, Salt Lake City, Utah, USA) and the Alexa488-conjugated goat-anti-chicken IgG (Invitrogen) were used as the primary and secondary antibodies for renal GFP staining. For COMP-Ang1 staining, the mouse monoclonal anti-FLAG-M2 IgG1 that specifically targets the FLAG tag of expressed COMP-Ang1 protein was applied as the primary antibody at $4^{\circ} \mathrm{C}$ for overnight, followed by incubating with the biotinSP-conjugated rat anti-mouse IgG for $30 \mathrm{~min}$ at room temperature and then detecting with the dichlorotriazinylamino fluorescein (DTAF)-conjugated-Streptavidin (Jackson ImmunoResearch, Laboratories) for $30 \mathrm{~min}$ at 
room temperature. To further identify the location of COMP-Ang1 in the glomeruli and pancreas, dual immunofluorescent staining for COMP-Ang1 and glomerular podocin or glomerular endothelial cells or pancreatic capillary endothelial cells was performed in AAVrh10. COMP-Ang1-treated mice. A mouse monoclonal antiFLAG-M2 IgG1 and a goat anti-podocin IgG (Santa Cruz Biotechnology, Santa Cruz, California, USA) / or a rat anti-mouse CD31 IgG2a (BD Biosciences, San Jose, California, USA) were applied as primary antibodies at the same time and kept at $4^{\circ} \mathrm{C}$ overnight. The rhodamine-Red ${ }^{\mathrm{TM}}$-x- conjugated donkey anti-goat IgG or $\mathrm{Cy}^{\mathrm{TM}} 3$-conjugated goat anti-rat IgG (Jackson ImmunoResearch) was applied as the secondary antibody for staining of glomerular podocytes or glomerular and pancreatic capillary endothelial cells at room temperature for 1 hour. After extensive washing, the cryostat sections were continuously incubated with the biotinSP-conjugated rat anti-mouse IgG and then detecting with the DTAF- conjugated-Streptavidin as described for staining of expressed COMP-Ang1 protein. In addition, to clarify whether COMP-Ang1 directly affects podocytes, dual immuno- fluorescent staining for Tie2 and podocin was also performed on frozen sections of kidney tissue. Similar to the dual-staining for COMP-Ang1 and glomerular podocytes, a mouse monoclonal anti-Tie2 IgG1k (Millipore) and a goat anti-podocin IgG were applied as primary antibodies and the same detecting antibodies were then used as described above. Dual-immunostained sections were analyzed using the Evos FL Auto2 Image System (Invitrogen). All control slides treated with PBS instead of primary antibodies showed no staining.

Immunofluorescent staining for fibronectin (FN), type IV collagen (Col IV), podocin, nephrin and Wilms tumor protein-1 (WT-1) was also performed on frozen sections as described previously. ${ }^{23}{ }^{24}$ Intraglomerular positive staining of FN, Col IV, podocin and nephrin was quantified separately in a blinded fashion by a computer-assisted method as described previously. ${ }^{25}$ The number of WT-1-positive podocyte per glomerulus was counted in 20 glomeruli selected randomly per section.

In addition, immunofluorescent staining for myeloperoxidase (MPO) to reflect the inflammatory status was also performed on $3 \mu \mathrm{m}$ cryostat pancreatic sections. A polyclonal goat-anti-human/mouse MPO IgG (R\&D Systems) was used as the primary antibody. The NorthernLight 557-conjugated donkey anti-goat IgG (R\&D Systems) was used as the secondary antibody. After washing, one drop of 4',6-diamidino-2-phenylindole (DAPI)-Fluoromount-G was applied on the pancreatic section. DAPI served as a fluorescent (ultraviolet) stain for cell nuclei. Islet positive staining of MPO was quantified by using Image J similar as analysis of glomerular staining.

\section{Western blot analysis}

Renal cortex tissue $(15 \mathrm{mg}$ ) from each mouse was homogenized in lysis buffer (Cell Signaling Technology, Beverly,
Massachusetts, USA) with $1 \%$ NP40, $1 \mathrm{mM}$ phenylmethylsulfonyl fluoride (PMSF) and one tablet $/ 5 \mathrm{~mL}$ protease inhibitor mix (Complete, Mini; Roche Diagnostics Corporation, Indianapolis, Indiana, USA). Equivalent amounts of protein pooled from individual mice within each group was processed for western blot analysis. Protein samples $(30 \mu \mathrm{g}$ each) were subjected to SDS-PAGE in $4 \%-12 \%$ gradient gel (Invitrogen) and immunoblotted on immobilon-P transfer membranes (Millipore Corporation, Bedford, Massachusetts, USA). Western blots of Ang1, Ang2, B-actin, plasminogen activator inhibitor-1 (PAI-1), FN, B7-1, desmin, phosphorylated-NFkB-p65, nicotinamide adenine dinucleotide phosphate (NAPDH) oxidase-2 (Nox2), and p47phox were assessed using rabbit anti-angiopoietin1 IgG (Millipore), rabbit anti-angiopoietin2 IgG (Millipore), mouse monoclonal anti-ß-actin, mouse monoclonal anti-PAI-1 IgG (BD Transduction Laboratories, USA), rabbit anti-human FN IgG, monoclonal rabbit-anti B7-1/CD80 IgG (OriGene Technologies, Rockville, Maryland, USA), mouse monoclonal anti-desmin IgG2a (Santa Cruz Biotechnology), mouse monoclonal anti-phospho-NF- kB-p65 IgG2b (Cell Signaling Technology), mouse anti-gp91(phox) (Nox2) IgG and mouse anti-p47(phox) IgG (BD Biosciences). Quantitation of the bands on autoradiograms was performed using a Bio-Rad GS-700 imaging densitometer (Bio- Rad Laboratories, Hercules, California, USA). Each protein level was normalized for the densitometric intensity of the glyceraldehyde 3-phosphate dehydrogenase (GAPDH) (using goat anti-GAPDH polyclonal antibody; GenScript, Piscataway, New Jersey, USA) or the B-actin. For comparison, this ratio was set at unity for normal control samples, and other lanes on the same gel were expressed as fold change over this value. All blots were run at least three times.

\section{RNA extraction and real time RT-PCR}

Total RNA was extracted from renal cortex tissue using Tri Reagent according to the manufacturer's instructions. Two micrograms of total RNA were reverse-transcribed using the superscript III first-strand synthesis system for RT-PCR kit (Invitrogen). Real- time RT-PCR was performed using the SYBR green dye I (Applied Biosystems, Foster City, California, USA) and the ABI 7900 Sequence Detection System (Applied Biosystems) as described previously. ${ }^{17}$ Samples were run as triplicates in separate tubes to permit quantification of the target gene normalized to GAPDH. Sequences of primers used for fibrotic markers were described previously. ${ }^{17}$ The specificity of the PCR products was double confirmed by $1.5 \%$ agarose gel by showing a specific single band with the expected size.

\section{Statistical analyses}

Data are expressed as mean \pm SD.D. with $\mathrm{n}$ representing the number of animals. The urinary MCP-1 values were $\log _{10}$-transformed prior to comparison to account for the skewed distribution. Groups were analyzed by one-way analysis of variance and subsequent Dunnett's test for multiple 
comparisons. $\mathrm{p}<0.05$ was considered statistically significant. The disease-induced increase in a variable was defined as the mean value for the disease control group minus the mean value of normal control group. The percentage of reduction in disease severity in a treated group was calculated as before. ${ }^{17}$

\section{RESULTS}

In vivo delivery of AAVrh10.GFP or AAVrh10.COMP-Ang1 to the mouse glomeruli and pancreas was optimal with intracarotid injection

As shown in figure $1 \mathrm{~A}$, the left carotid artery injection of AAVrh10.GFP showed a similar or even better glomerular targeting in the kidney when compared with the direct renal delivery of AAVrh10.GFP. There was no or very limited trace of local GFP protein found in the kidney of the mice receiving tail vein injection of AAVrh10.GFP, but extensive GFP protein was found in the lungs of these mice (data not shown). The method of the artery injection of AAVrh10.GFP is also more practical and imminently translatable to clinical trials, compared with direct renal delivery. Based on these results, in vivo delivery of AAVrh10.COMP-Ang1 to glomeruli through the left carotid artery injection was chosen as the optimal route delivery of AAVrh10. COMP-Ang1 to the kidney in the following experiment.

As expected, diabetic $\mathrm{db} / \mathrm{db}$ mice treated with a single dose of AAVrh10.COMP-Ang1 overexpressed COMP-Ang1 in diabetic kidney tissue, as shown in figure 1B. Furthermore, COMP-Ang1 protein which was labeled with FLAG (green) was mainly detected in renal glomeruli by immunofluorescent staining (figure 1C). No COMP-Ang1 was found in untreated diabetic $\mathrm{db} /$ $\mathrm{db}$ mice or non-diabetic $\mathrm{db} / \mathrm{m}$ (data not shown). The results of dual immunostaining are shown in figure 1D. COMP-Ang1 stained in green (FLAG) and CD31 stained in red (CD31) were merged and produced clear yellow staining (FLAG/CD31), while COMP-Ang1 (FLAG in green) and podocin (in red) did not, indicating that COMP-Ang1 was partially colocalized with glomerular endothelial cells, not podocytes. It remains unknown whether COMP-Ang1 is colocalized with glomerular mesangial cells since there are no proper antibodies available for staining of mesangial cells when the mouse monoclonal anti-FLAG-M2 IgG1 is applied at the same time. In addition, Tie2 (green) was extensively stained in glomeruli in non-diabetic $\mathrm{db} / \mathrm{m}$ mice, which was reduced in many glomeruli in $\mathrm{db} / \mathrm{db}$ mice at 18 weeks of age as shown in figure $1 \mathrm{E}$. However, diabetes-induced reduction of glomerular Tie2 staining was partially ameliorated in AAVrh10.COMP-Ang1-treated $\mathrm{db} / \mathrm{db}$ mice (figure $1 \mathrm{E}, \mathrm{db} / \mathrm{db}+\mathrm{COMP}-\mathrm{Ang} 1$ ). The results of dual immunostaining revealed that the majority of Tie2 (green) was existed internal to podocyte foot processes, which were labeled with podocin (red). A small portion of Tie2 might be colocalized with podocytes shown in a few yellow color (figure 1E). These results indicate that injected AAVrh10.COMP-Ang1 mainly targets glomerular endothelial cells (and possible mesangial cells) by expressing COMP-Ang1 and/or binds to endothelial cells via the Tie2 receptor. Whether elevated COMP-Ang1 protects directly against podocyte injury needs to be further identified. Nonetheless, consistent with renal mRNA expression and protein staining of COMP-Ang1, treatment with AAVrh10.COMP-Ang1 had no effect on Ang2 production but effectively ameliorated diabetes-induced reduction of total Ang1 and the abnormal ratio of Ang2/Ang1 in diabetic renal cortex tissue, as shown in figure 1F. Surprisingly, expression of COMP-Ang1 was also detected in the pancreas in treated $\mathrm{db} / \mathrm{db}$ mice as shown in figure $1 \mathrm{G}$, but not in the untreated $\mathrm{db} / \mathrm{m}$ or $\mathrm{db} / \mathrm{db}$ mice. The COMP-Ang1 protein (FLAG in green) was primarily expressed in pancreatic islet capillary endothelial cells, which were labeled with CD31 (red), as evidenced by the colocalization of FLAG stained in green and CD31 stained in red that yielded yellow color when both of them were merged, as shown in figure $1 \mathrm{H}$.

\section{Effect of AAVrh10.COMP-Ang1 in diabetic $\mathrm{db} / \mathrm{db}$ mice AAVrh10.COMP-Ang1 reduces hyperglycemia and albuminuria}

The characteristics of the three groups of mice are presented in figure 2; body weights of $\mathrm{db} / \mathrm{db}$ mice were greater than those of $\mathrm{db} / \mathrm{m}$ controls. Treated and untreated $\mathrm{db} / \mathrm{db}$ mice showed no difference in body weight. The untreated $\mathrm{db} / \mathrm{db}$ mice maintained high levels of BG with significantly elevated HbAlc levels. Accordingly, daily water intake and urine output were notably increased in $\mathrm{db} / \mathrm{db}$ mice (data not shown). Remarkably, treatment with AAVrh10.COMP-Ang1 yielded a significant reduction of glycaemia in $\mathrm{db} / \mathrm{db}$ mice starting at 2 weeks and lasting for 8 weeks after treatment, and led to a clinically meaningful reduction in $\mathrm{HbA1c}$ levels from $11.3 \%$ to $7.2 \%$, compared with untreated $\mathrm{db} / \mathrm{db}$ mice measured at 18 weeks of age.

Plasma BUN and Cr concentrations of diabetic $\mathrm{db} / \mathrm{db}$ mice were identical to those of the non-diabetic $\mathrm{db} / \mathrm{m}$ mice. Treatment with AAVrh10.COMP-Ang1 had no effect on plasma BUN and Cr levels. These results may indicate that $\mathrm{db} / \mathrm{db}$ mice at 18 weeks of age had not yet developed uremia.

By measuring 24 hours urinary albumin excretion (UAE), we found that diabetic $\mathrm{db} / \mathrm{db}$ mice secreted more urinary albumin than $\mathrm{db} / \mathrm{m}$ mice starting 2 weeks after the uninephrectomy (10 weeks of age). At 12 weeks of age, the UAE levels in $\mathrm{db} / \mathrm{db}$ control mice were markedly higher than those in $\mathrm{db} / \mathrm{m}$ controls. Subsequent levels of UAE were continuously elevated until 18 weeks of age in $\mathrm{db} / \mathrm{db}$ mice, consistent with our previous reports. ${ }^{26}$ AAVrh10.COMP-Ang1 treatment was thus administered to $\mathrm{db} / \mathrm{db}$ mice at 10 weeks of age, before onset of albuminuria. Of note, this treatment dramatically slowed the progressive increases in albuminuria by $70 \%$ in $\mathrm{db} / \mathrm{db}$ mice. 

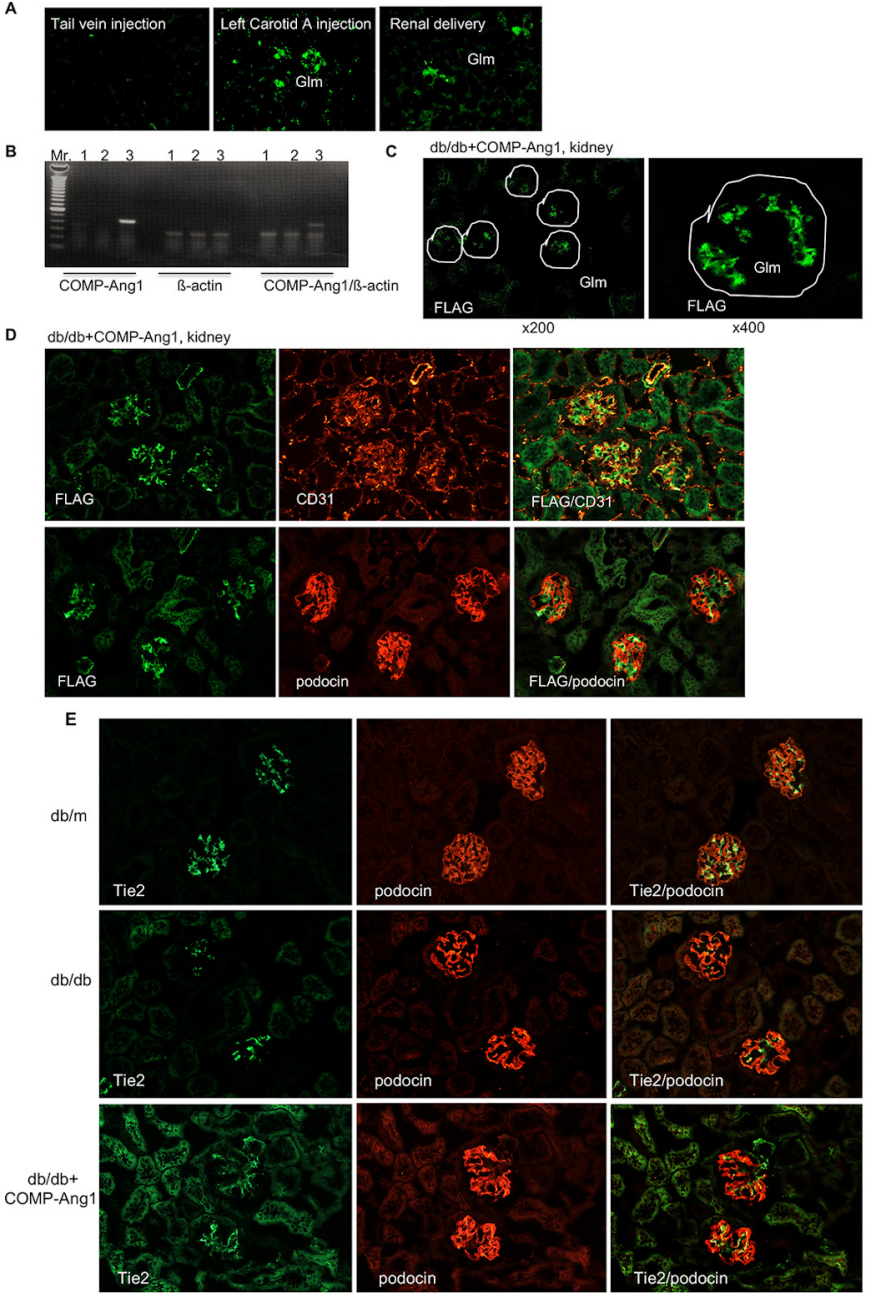

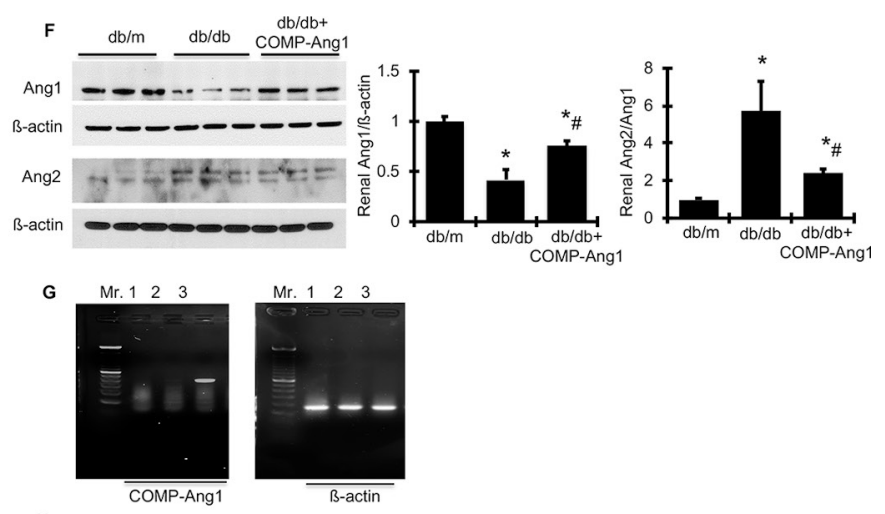

H db/db+COMP-Ang1, pancreas
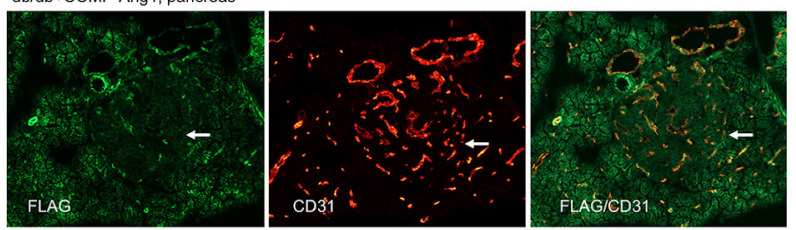

Figure 1 In vivo delivery of AAVrh10.COMP-Ang1. (A) Intracarotid injection delivers AAV10rh.green fluorescent protein (GFP) to the kidney. Representative renal photomicrographs were from normal mice 2 weeks after receiving either tail vein injection $(n=2)$, left carotid arterial injection $(n=2)$, or direct renal delivery of AAVrh10.GFP with a mini-pump ( $n=2)$. GFP protein in the kidney was detected by immunofluorescent staining using chicken- anti-GFP antibody and Alexa-488 goat anti-chicken IgG. Magnification: $\times 200$. $(B, G)$ Semiquantitative RT-PCR demonstrated that COMP-Ang1 was expressed in the kidney $(B)$ and the pancreas $(\mathrm{G})$ of $\mathrm{db} / \mathrm{db}$ mice injected with AAVrh10.COMP-Ang1. Mr. DNA size marker, 1, renal cortex RNA from db/m. 2, db/db mice, and 3, AAVrh10.COMP-Ang1-treated db/db mice. B-actin mRNA expression served as a control. (C) Immunofluorescent staining for COMP-Ang1 protein in the kidney using the monoclonal anti-FLAG-M2 IgG1 since the pcDNA vector contains a FLAG tag expression. Representative renal photomicrographs from treated db/db mice at 8 weeks after receiving intracarotid arterial injection of AAVrh10.COMP-Ang1(db/db+COMP-Ang1). Magnification: $\times 200$ or $\times 400$ for kidneys. (D) Dual immunofluorescent staining for glomerular COMP-Ang1, which was labeled with FLAG (green); glomerular endothelial cells, which were labeled with CD31 (red); and dual immunofluorescent staining for glomerular COMP-Ang1 (green) and glomerular podocyte food processes, which were labeled with podocin (red). Representative renal photomicrographs from treated db/db mice at 8 weeks after receiving intra-carotid arterial injection of AAVrh10.COMP-Ang1 (db/db+COMP-Ang1). Magnification, $\times 200$. (E) Dual immunofluorescent staining for glomerular Tie2 (green) and glomerular podocytes (podocin stained in red). Representative renal photomicrographs from non-diabetic $\mathrm{db} / \mathrm{m}$, untreated db/db mice and AAVrh10.COMP-Ang1-treated db/ $\mathrm{db}$ mice $(\mathrm{db} / \mathrm{db}+$ COMP-Ang1) at 18 weeks of age. Magnification, $\times 200$. (F) Representative western blots illustrating Ang 1, Ang2 and $B$-actin protein expression in renal cortical tissues. The graphs summarize the results of band density measurements for Ang1 and the ratio of Ang1/Ang2 (shown on the right). *versus db/m ( $n=6), p<0.05$; \#versus db/db $(n=6), p<0.05 . d b /$ $\mathrm{db}+$ COMP-Ang1, $\mathrm{n}=5$. (H) Dual immunofluorescent staining for pancreatic COMP-Ang1 (FLAG stained in green) and capillary endothelial cells (CD31 stained in red). Representative pancreas photomicrographs from treated db/db mice at 8 weeks after receiving intracarotid arterial injection of AAVrh10.COMP-Ang1 (db/db+COMP- Ang1). Magnification, $\times 200$. The arrows indicate an islet.

\section{AAVrh10.COMP-Ang1 reduces renal fibrosis and renal podocyte injury}

Representative glomeruli stained with PAS are shown in figure $3 \mathrm{~A}, \mathrm{~B}$. At the study endpoint, the glomeruli of 18-week-old $\mathrm{db} / \mathrm{db}$ mice demonstrated increased glomerulosclerosis relative to non-diabetic controls, 

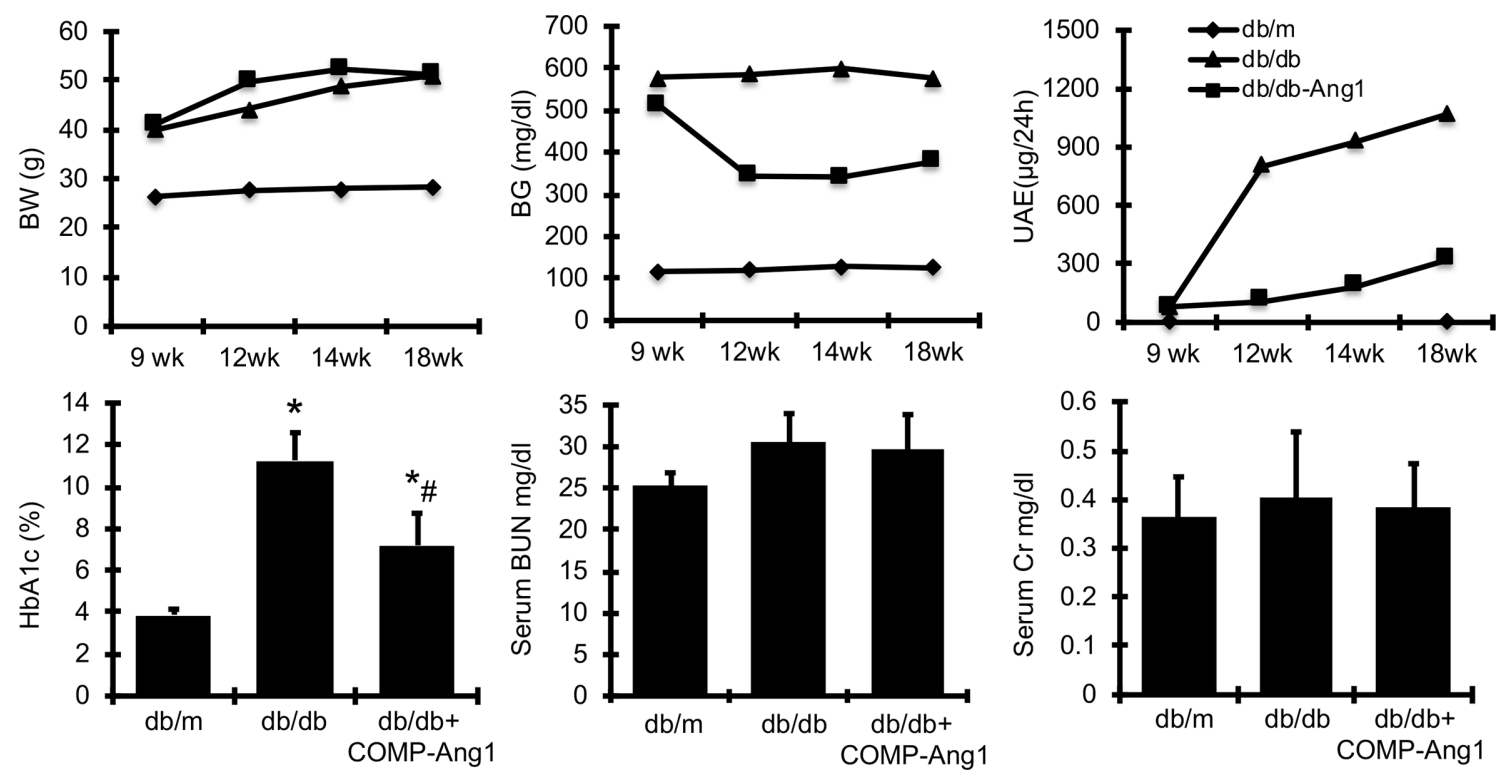

Figure 2 AAVrh10.COMP-Ang1 improves systemic and renal clinical parameters of diabetic $\mathrm{db} / \mathrm{db}$ mice. *versus $\mathrm{db} / \mathrm{m}$ ( $\mathrm{n}=6$ ), $\mathrm{p}<0.05$; \#vs db/db $(n=6), p<0.05$. BG, blood glucose; BUN, blood urea nitrogen; BW, body weight; Cr, creatinine; HbA1c, glycosylated hemoglobin; UAE, urinary albumin excretion.

with considerable accumulation of PAS-positive pink extracellular matrix (ECM) proteins as quantified by computer-assisted color image analysis of the glomerular mesangium. We further assessed protein localization of FN and Col IV in glomeruli, two major components of glomerular ECM. Consistent with the accumulation of glomerular PAS- positive ECM in $\mathrm{db} / \mathrm{db}$ mice, immunofluorescently labeled glomerular FN and Col IV was markedly increased in $\mathrm{db} / \mathrm{db}$ mice at week 18 . However, treatment with AAVrh10.COMP-Ang1 reduced the ECM accumulation in diabetic glomeruli by $61 \%$. Surprisingly, this treatment completely reversed glomerular deposition of FN and Col IV stimulated by diabetes, similar to those levels in non-diabetic normal controls.

As a key modulator of matrix accumulation, PAI-1 plays an important role in decreased turnover of pathological ECM. ${ }^{27}$ As expected, renal PAI-1 mRNA levels were increased by 2.54 -fold in $\mathrm{db} / \mathrm{db}$ mice, compared with that of $\mathrm{db} / \mathrm{m}$ controls (figure $3 \mathrm{~F}$ ).

Similarly, renal FN and Col IV mRNA levels were increased in $\mathrm{db} / \mathrm{db}$ mice, compared with normal controls (figure $3 \mathrm{G}, \mathrm{H}$ ). This rise in mRNA expression was consistent with increased protein levels of PAI- 1 and FN in western blots of renal cortex tissue (figure 3C-E). However, both the mRNA expression and the protein production levels of these fibrotic markers in renal cortex induced in $\mathrm{db} / \mathrm{db}$ mice were decreased significantly in AAVrh10.COMP-Ang1-treated $\mathrm{db} / \mathrm{db}$ mice (figure 3C-H).

As shown in figure 3I,J detected by immunofluorescent staining, non-diabetic $\mathrm{db} / \mathrm{m}$ mice showed intense and lining staining for nephrin and podocin, two podocyte slit diaphragm-associated proteins, along the capillary in glomeruli. In contrast, diabetes resulted in an attenuation of staining by $25.4 \%$ for podocin and $42.8 \%$ for nephrin $(\mathrm{p}<0.05)$ in the glomeruli in $\mathrm{db} / \mathrm{db}$ mice at 18 weeks of age, which was largely restored by AAVrh10.COMP-Ang1 treatment.

WT-1 is a nuclear protein specific to podocytes and parietal glomerular epithelial cells in the adult kidney. ${ }^{28}$ WT-1-positive cells within the glomerular area were counted. Consistent with the staining of podocin and nephrin, glomeruli from $\mathrm{db} / \mathrm{db}$ mice contained fewer podocytes than did glomeruli from non-diabetic $\mathrm{db} / \mathrm{m}$ mice, as illustrated by immunofluorescent staining in figure 3I,J. However, AAVrh10. COMP-Ang1-treated $\mathrm{db} / \mathrm{db}$ mice had increased numbers of podocytes compared with untreated $\mathrm{db} / \mathrm{db}$ mice.

Protein levels of desmin, a conventional marker for podocyte injury, were upregulated by $407 \%$ in the renal cortex of diabetic $\mathrm{db} / \mathrm{db}$ mice $(\mathrm{p}<0.05$, figure $3 \mathrm{~K}, \mathrm{~L})$. AAVrh10.COMP-Ang1 treatment reversed this diabetesinduced increase. The protein B7-1 can serve as a podocyte injury marker in response to split diaphragm protein rearrangement and foot process effacement. ${ }^{30}$ As shown in figure $3 \mathrm{~K}, \mathrm{M}, \mathrm{B} 7-1$ protein levels were markedly increased in diabetic renal tissue in $\mathrm{db} / \mathrm{db}$ mice. Although elevated production of desmin and B7-1 was not specifically confirmed in podocytes, the changes of both desmin and B7-1 were consistent with the changes of the two slit- diaphragm-associated proteins, nephrin and podocin, suggesting that diabetic $\mathrm{db} /$ $\mathrm{db}$ mice sustain injury to podocytes. Consistent with improvement of podocin and nephrin levels, AAVrh10. COMP-Ang1 treatment also successfully reduced the elevated $\mathrm{B} 7-1$ protein expression in $\mathrm{db} / \mathrm{db}$ mice by $77.5 \%(\mathrm{p}<0.05)$.

Collectively, these data suggest that treated diabetic $\mathrm{db} / \mathrm{db}$ mice have ameliorated podocyte dysfunction as evidenced by reduced podocyte loss and preserved expression of slit-diagram proteins, which may result in 

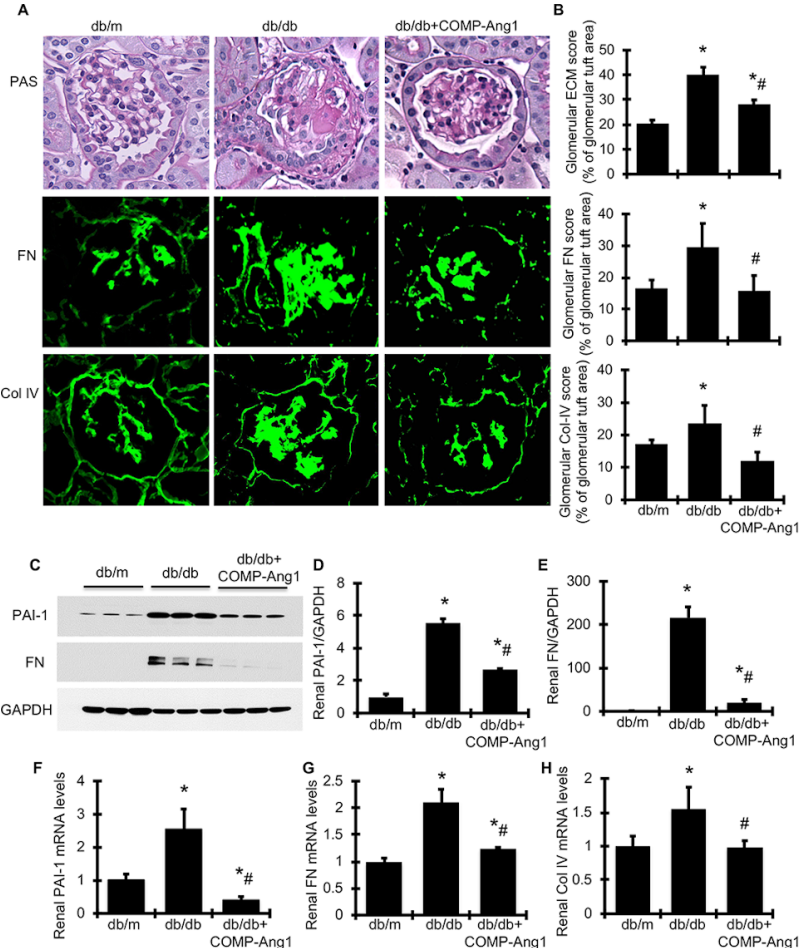
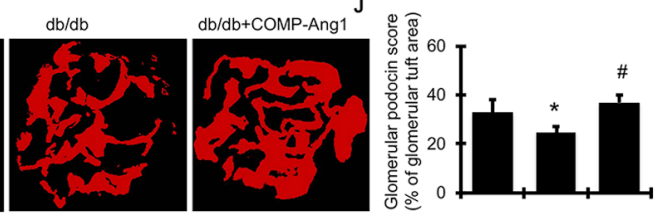

nephrin
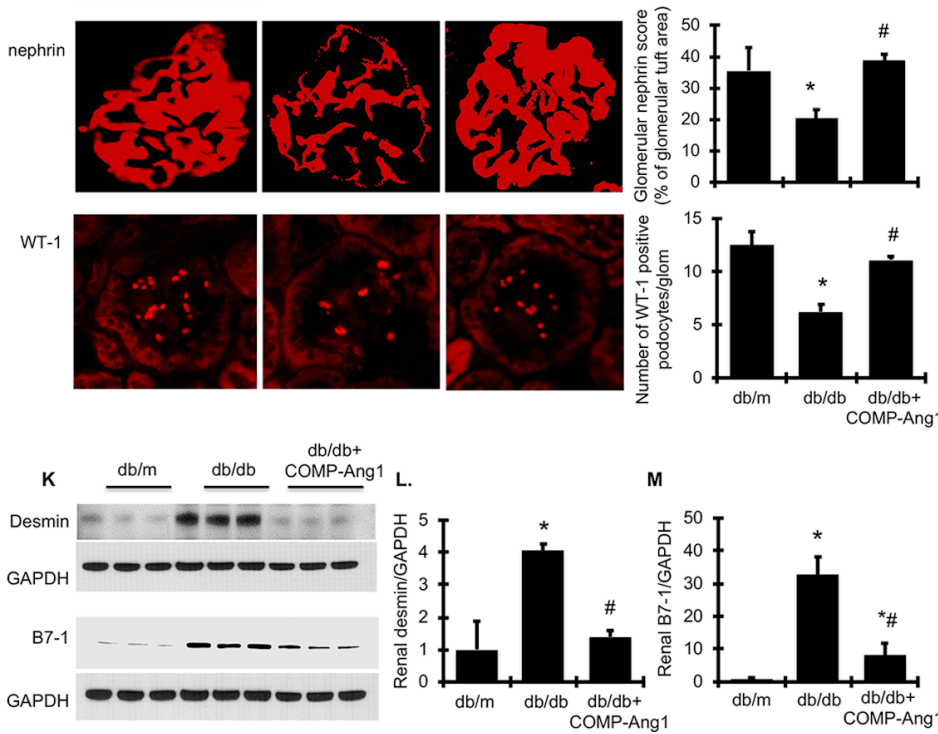

Figure 3 AAVrh10.COMP-Ang1 reduces glomerulosclerosis and podocyte injury in diabetic $\mathrm{db} / \mathrm{db}$ mice. (A) The histological sections stained with PAS, or FN, or Col IV are presented at $\times 400$ magnification. (B) Graphic representations of glomerular staining score. (C) Representative western blots illustrating PAI-1, FN and GAPDH protein expression in renal cortical tissues. The graphs summarize the results of band density measurements for PAl-1 (D) and FN (E). (F) Expression of PAl-1 mRNA. (G) Expression of FN. (H) Expression of type $\alpha 1$ (IV) collagen mRNA. Expression of mRNA was determined by real-time RT/PCR. Changes in mRNA levels were determined by first correcting the amplification of GAPDH for each sample. (I) Representative photomicrographs (at original magnification $\times 400$ ) of glomeruli from normal control mice $(\mathrm{db} / \mathrm{m}, \mathrm{n}=6$ ), diabetic $\mathrm{db} / \mathrm{db}$ mice without treatment at week $18(n=6)$ or treated with AAVrh10.COMP-Ang1 (db/db+COMP-Ang1, $n=5)$. (J) Graphic representation of glomerular podocin and nephrin staining scores or glomerular number of WT-1 positive podocytes was shown. (K)

Representative western blots illustrating desmin, B7-1, and GAPDH protein expression in renal cortical tissues. The graphs summarize the results of band density measurements for desmin $(L)$ and B7-1 (M). *versus $\mathrm{db} / \mathrm{m}(n=6), p<0.05$; \#versus $\mathrm{db} /$ $\mathrm{db}(\mathrm{n}=6), \mathrm{p}<0.05$. Col IV, type IV collagen; ECM, extracellular matrix; FN, fibronectin; GAPDH, glyceraldehyde 3-phosphate dehydrogenase; PAI-1, plasminogen activator inhibitor; PAS, periodic acid-Schiff; WT-1, Wilms tumor protein-1.

a significant decrease in proteinuria and remission of glomerulosclerosis.

\section{AAVrh10.C0MP-Ang1 reduces oxidative stress markers involved in the progression of DN}

To verify the renoprotective effect of overexpression of COMP-Ang1 on related cellular signaling pathways, we further observed that protein production of the Nicotinamide adenine dinucleotide phosphate (NAPDH) oxidase family members, Nox2 and its regulatory subunit, $\mathrm{p} 47^{\text {phox }}$, was much greater in the renal cortex of diabetic $\mathrm{db} / \mathrm{db}$ mice compared with non-diabetic $\mathrm{db} / \mathrm{m}$ mice (figure $4 \mathrm{~A}-\mathrm{C}$ ), indicating significant activation of Nox2 in diabetic kidney tissue. In parallel with renal activation of NAPDH oxidase, urine MDA content, the most prevalent by-product of renal oxidative stress, was consistently and dramatically increased in $\mathrm{db} / \mathrm{db}$ mice compared with $\mathrm{db} / \mathrm{m}$ control mice (figure 4D). However, all of these markers of oxidative stress were markedly reduced to or close to normal levels in AAVrh10.COMP-Ang1-treated $\mathrm{db} / \mathrm{db}$ mice. Likewise, increased renal production of p- NF-kB-p65 and urine production of MCP- 1 in $\mathrm{db} / \mathrm{db}$ mice was significantly reduced by AAVrh10.COMP-Ang1 treatment (figure 4E,F). In total, these data indicate that overexpression of COMP-Ang1 inhibits renal NF-kBmediated inflammation and NAPDH oxidase-mediated oxidative stress in $\mathrm{db} / \mathrm{db}$.

\section{AAVrh10.COMP-Ang1 increases islet beta cell staining in pancreatic tissue in $\mathrm{db} / \mathrm{db}$ mice}

As AAVrh10.COMP-Ang1 yielded a significant reduction of glycemia in $\mathrm{db} / \mathrm{db}$ mice, this strongly indicated that a single dose of our sustained treatment could impact microvascular dysfunction and insulin generation and action in pancreatic islets (or insulin-targeted organs) beyond the kidneys. We therefore examined pancreatic metrics of diabetes and observed that delivery of AAVrh10. COMP-Ang1 reduced diabetes-induced islet hyperplasia and improved $B$-cell secretory function in $\mathrm{db} / \mathrm{db}$ mice as determined by increased $B$-cell density (figure $5 \mathrm{~A}$ ) and circulating insulin levels $(6.84 \pm 1.21$ vs $2.39 \pm 0.28, \mathrm{ng} / \mathrm{mL}$, $\mathrm{p}<0.05)$. Such amelioration in B-cell function expectedly yielded a significant reduction of glycemia in treated $\mathrm{db} /$ db mice. 


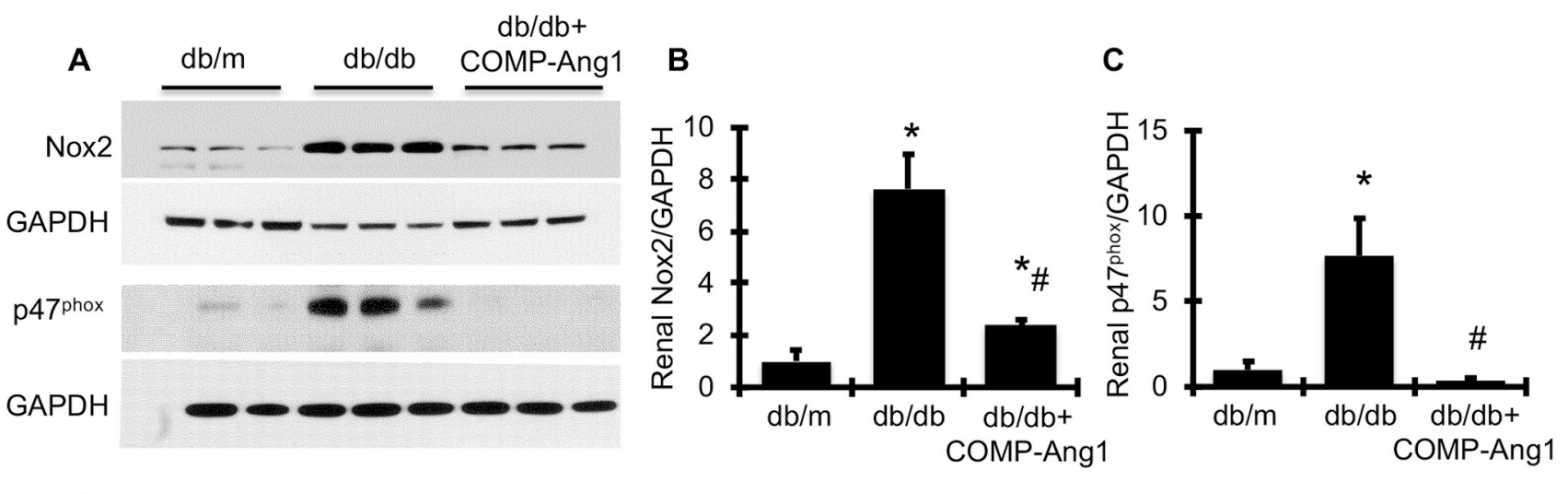

D

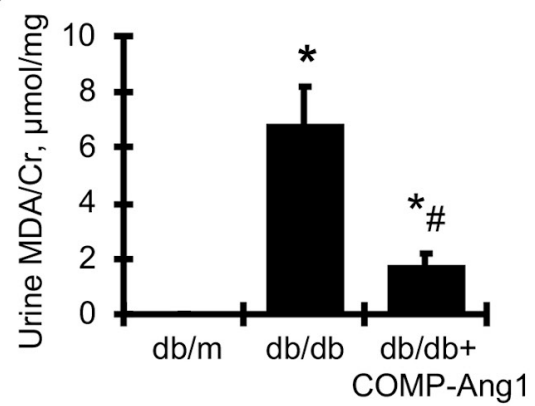

E
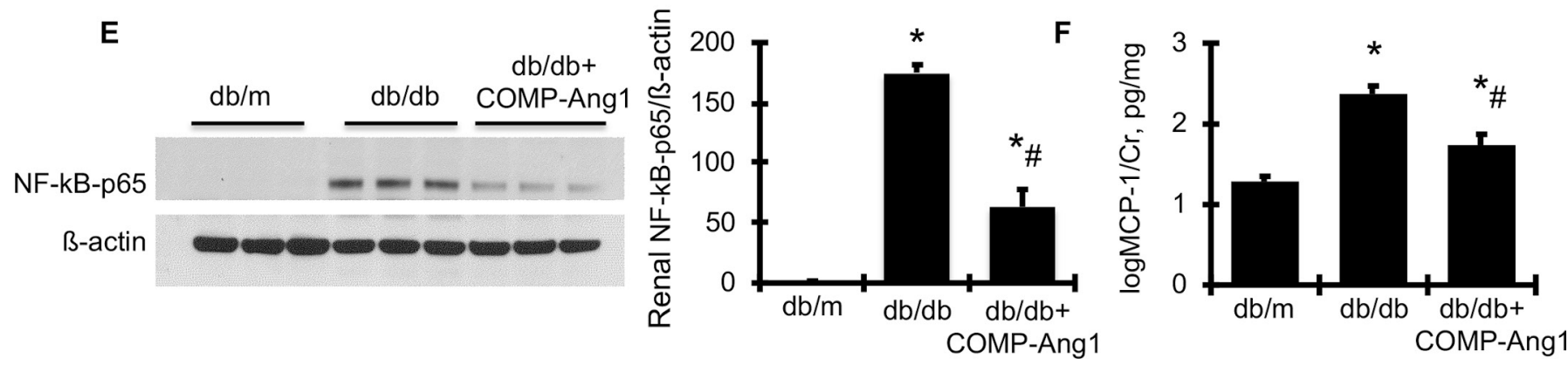

Figure 4 AAVrh10.COMP-Ang1 reduces renal oxidative stress and related renal information markers in diabetic $\mathrm{db} / \mathrm{db}$ mice. (A) Representative western blots illustrating Nox2 and GAPDH protein and $\mathrm{p} 47^{\text {phox }}$ and GAPDH expression in renal cortical tissues. The graphs summarize the results of band density measurements for Nox2 (B) and p4 $7^{\text {phox }}$ (C). (D) Urinary MDA excretion levels. (E) Representative western blots illustrating NF-kB-p65 and B-actin protein expression in renal cortical tissues. Quantification of the band density measurements is shown beside the blots. (F) Urinary MCP-1 excretion levels. *versus $\mathrm{db} / \mathrm{m}$ $(n=6), p<0.05$; \#versus db/db ( $n=6), p<0.05$. Cr, creatinine; GAPDH, glyceraldehyde 3-phosphate dehydrogenase; MCP-1, monocyte chemoattractant protein-1; MDA, malondialdehyde; NAPDH, nicotinamide adenine dinucleotide phosphate; Nox2, NAPDH oxidase-2.

\section{AAVrh10.COMP-Ang1 reduces inflammation in pancreatic tissue in $\mathrm{db} / \mathrm{db}$ mice}

Inflammation of the pancreas has been suggested to play a prominent pathogenetic role in the development or progression of type 2 diabetes. MPO is mainly expressed in neutrophils and usually used as a biomarker of activated neutrophils. Therefore, immunostaining for MPO of pancreatic tissue was performed to assess the condition of tissue neutrophil infiltration. As shown in figure 5B,C, the immunofluorescent staining of MPO indicated that $\mathrm{db} / \mathrm{db}$ mice experienced increased neutrophil infiltration in pancreatic tissue compared with $\mathrm{db} / \mathrm{m}$ control mice at 18 weeks of age. However, treatment with AAVrh10.COMP-Ang1 largely retarded the increased MPO-positive neutrophil infiltration in pancreatic tissue in $\mathrm{db} / \mathrm{db}$ mice. These data indicate that
AAVrh10.COMP-Ang1 treatment suppresses components of diabetes-mediated inflammation associated with both kidney and pancreas.

\section{DISCUSSION}

We demonstrated herein a protective effect of localized COMP-Ang1 expression on the progression of glomerular and islet B-cell injury, using a type 2 diabetic mouse model with pathological features resembling type 2 diabetic patients. Using the AAVrh10 serotype, we found significant improvement of the microvasculature in the kidney and in the pancreas consistent with observed improvements in diabetic retinopathy after intravitreal injection of AAV2.COMP-Ang $1,{ }^{19}$ indicating that reinstating hyperglycemia-induced reduction of Tie2 via 


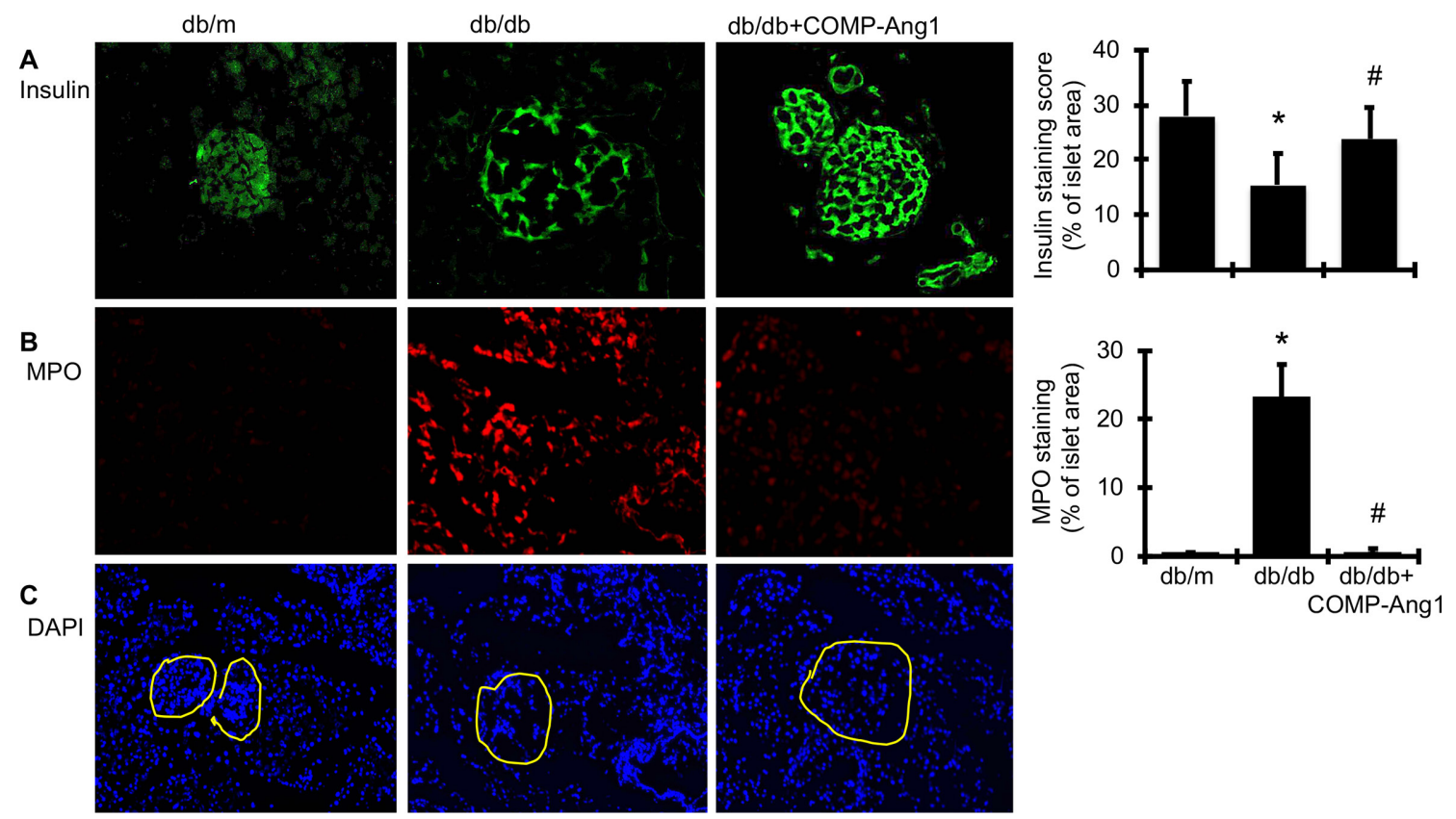

Figure 5 AAVrh10.COMP-Ang1 increases pancreatic B-cell density and reduces pancreatic neutrophil infiltration in diabetic $\mathrm{db} / \mathrm{db}$ mice. (A) Representative photomicrographs (at original magnification $\times 400$ ) of pancreatic tissue immunofluorescently stained with insulin antibody from normal control mice $(\mathrm{db} / \mathrm{m})$, diabetic $\mathrm{db} / \mathrm{db}$ mice without treatment at week 18 and diabetic $\mathrm{db} / \mathrm{db}$ mice treated with AAVrh.COMP-Ang1 (db/db+COMP-Ang1) and the graphic representations of islet insulin staining score are shown. (B) Reduced pancreatic neutrophil infiltration as evidenced by decreased MPO staining (in red). 4',6-diamidino-2phenylindole (DAPI) (C, in blue) stained nuclei served as cell counterstaining of MPO staining (at original magnification $\times 200$ ). Graphic representations of islet staining score of MPO are shown on the right. *versus $\mathrm{db} / \mathrm{m}(\mathrm{n}=6)$, $p<0.05$; \#versus $\mathrm{db} / \mathrm{db}$ $(n=6), p<0.05$. MPO, myeloperoxidase.

local Ang1 production is sufficient for maintaining the integrity of microvasculature in major organs associated with diabetes. AAVrh10 (as compared with AAV2 used for intravitreal injection) was chosen as it has superior tropism to kidney and visceral organs, low reported human immune response within the human population and high capacity for sustained expression, ${ }^{22}$ thus eliminating the need for repeated delivery. Critically, arterial delivery of AAV-based drug therapies to improve targeting and localization holds significant clinical feasibility for human patients suffering from diabetic kidney and pancreatic diseases. Prior work by Lee et al revealed that repeated systemic COMP-Ang1 administration (through tail vein injection every other week) using recombinant adenoviral delivery (Ade-COMP-Ang1) also ameliorated disease progression such as albuminuria and glomerulosclerosis in type $2 \mathrm{DN}$ in mice ${ }^{21}$ by increasing circulating serum levels of COMP-Ang1. However, when we compared the efficacy of intracarotid artery versus tail vein injections of AAVrh10.COMP-Ang1, both treatments led to similar elevated circulating serum levels of COMP-Ang1 (see online supplementary figure 1A), but reduction of disease severity was significantly enhanced in mice injected via the carotid artery (see online supplementary figure 1B). Of note, no reduction in BG levels and HbA1c levels was observed in $\mathrm{db} / \mathrm{db}$ mice receiving tail vein injection of AAVrh10.COMP-Ang1 (see online supplementary figure $2 \mathrm{~A}, \mathrm{~B}$ ). It remains to be seen whether increasing the IV injection dose of AAV.
COMP-Ang1 will increase its therapeutic effect in $\mathrm{db} / \mathrm{db}$ mice. As previous studies using recombinant adenovirus to express COMP-Ang1 had no effect on serum insulin levels, ${ }^{21}$ it is unlikely that intravenous treatment ameliorated pancreatic microvasculature. In contrast, our study suggests that sustained gene therapy with a single arterial AAV injection directed to the visceral organs can provide significant benefits to both renal and pancreatic function, with great translational potential for human disease. The larger size of human patients will enable interventional radiology techniques, further simplifying vector delivery to the target area.

As expected, AAVrh10.COMP-Ang1-mediated markers of oxidative stress, inflammation, and fibrosis in diabetic kidneys in line with improved glomerular endothelial integrity and function. Based on the dual staining data of COMP-Ang1/podocin, it is less possible that the amelioration of podocyte injury after treatment with AAVrh10. COMP-Ang1 in $\mathrm{db} / \mathrm{db}$ mice is due to a direct effect of COMP-Ang1 on podocytes. Alternatively, the amelioration of glomerular signal pathways of inflammation and oxidative stress after treatment may ameliorate podocyte injury. These results further confirmed that sustained, localized long-term replacement of Ang1 in the early phase of DN slows the progression of DN.

The islet endothelial cells of pancreatic islet capillaries not only are involved in the delivery of oxygen and nutrients to $\beta$-cells, but induce insulin gene expression during islet development, modulate adult $\beta$-cell function, 
promote B-cell proliferation, and produce a number of vasoactive substances and growth factors. ${ }^{31}{ }^{32}$ Abnormal islet capillary morphology, including endothelial cell loss, vascular disruption, thickening and fragmentation of islet capillaries, has been observed in both human and animal pancreases from individuals with T2DM. ${ }^{33-36}$ Islet endothelial degeneration may thus contribute to the severity or progressive islet failure in T2DM. However, the causal mechanism of diabetes-associated degeneration of the islet endothelia remains to be elucidated, as does the role of Ang1-Tie2 signaling within islet vasculature, and the contribution of defective islet Ang1-Tie2 signaling to diabetic disease progression.

Ang1 is normally expressed mainly by perivascular cells and B-cells in mouse and human islets, ${ }^{37}$ whereas its receptor is expressed on endothelial cells. Although endothelial Ang2 expression generally antagonizes Tie2 signaling, the dynamics of this process have not been clarified with respect to the pancreatic islets, particularly under diabetic conditions. However, under normal conditions, it has been shown that B-cell- specific overexpression of Ang1 or Ang2 only slightly impairs insulin secretory output and glucose tolerance together with marginal altered vascularization, islet mass and morphology. ${ }^{38}$ A recent study showed that B-cell-specific Ang2 overexpression in mice led to hypovascularized islets, increased apoptosis, and reduced B-cell mass in response to a high fat diet but had no major influence on glucose homeostasis. ${ }^{39}$ Meanwhile, a defect in B-cellderived Ang1 induced islet inflammation, disrupted the normal intraislet vascular ultrastructure and impaired the insulin secretory mechanism in response to a high fat diet. ${ }^{40}$ Although the mouse high fat diet model differs from human patients with respect to the development of T2DM, these reports nonetheless indicate that the balance of Ang1 and Ang2 governs the microenvironment and function of islets under metabolic challenge. Importantly, the dysregulation of the secretion of Ang1/2 equilibrium by either decreased Ang1 or increased Ang2 levels has been clinically associated with T2DM and vascular dysfunction, ${ }^{91}$ underscoring the question of whether altered Ang1-Tie2 signaling contributes to impaired pancreatic islet vasculature, homeostasis and diabetes progression. A limitation of the present study was our inability to directly assay Ang1-Tie2 signaling in islets in $\mathrm{db} / \mathrm{db}$ mice. Nevertheless, COMP-Ang1 treatment is clearly islet protective in these mice, as indicated by significantly increased B-cell density, improved B-cell secretory function and reduced pancreatic inflammation, contributing to suppression of hyperglycemia. These results also suggest that altered Ang1-Tie2 signaling may occur in pancreatic islets as it does in glomeruli in $\mathrm{db} / \mathrm{db}$ mice. To our knowledge, the present study is the first evidence implicating susceptibility of Ang1-Tie2 signaling within pancreatic vasculature to type 2 diabetic hyperglycemia (similarly to renal glomeruli), as well as the first to show that accessory Ang1-Tie2 signaling may promote pancreatic islet homeostasis in diabetes. Previously, COMP-Ang1 treatment was shown to ameliorate the abnormal metabolic homeostasis of epididymal adipose tissue in both type 1 and type 2 diabetic mouse models. ${ }^{21}{ }^{42}$ Further studies will determine whether treatment with AAVrh10. COMP-Ang1 improves insulin resistance in insulintargeted organs in type 2 diabetes. Nonetheless, it is likely that reduced glucose load or glucose toxicity may, at least partially, account for the observed reduction of renal podocyte injury, PAI- 1 synthesis, NAPDH oxidasemediated oxidative stress and inflammatory biomarkers in $\mathrm{db} / \mathrm{db}$ mice.

In summary, our success in delivery of AAVrh10. COMP-Ang1 to pancreatic islet capillaries and renal glomerular endothelial cells demonstrates that activation of Tie2 signaling by sustained, localized long-term replacement of Ang1 promotes vascular normalization in diabetic visceral microangiopathy affecting both the pancreas and the kidney in T2DM. The potential of activating Ang1-Tie2 signaling for reducing hyperglycemia and glomerulosclerosis in $\mathrm{db} / \mathrm{db}$ mice suggests that AAVrh10.COMP- Ang1 holds promise as a powerful new gene therapy approach for treating or reversing type 2 diabetes and the progression of type $2 \mathrm{DN}$.

Contributors MT, LSC and LT performed the experimental study, sample collection and analyses of samples. LT edited the manuscript. HU constructed the vector of the plasmids and measured plasma COMP-Ang1 levels. CW helped design and discuss the study. BKA designed the study, analysed the results and edited the manuscript. YH designed and performed the experimental study, sample collection and analyses of samples; wrote and edited the manuscript; and is the guarantor of this work and, as such, had full access to all the data in this study and takes responsibility for the integrity of the data and the accuracy of the data analysis. All authors approved the manuscript.

Funding This work was supported by the American Heart Association (No. 16GRNT27610030) $(\mathrm{YH})$ and the American Diabetes Association (number 1-17-IBS312) (YH). This work was also partially supported by NEI R01 EY026029 (BKA) and RPB Unrestricted Grant (BKA). LT was the recipient of postdoctoral fellowship grant from Center of Kidney Transplantation, Ningbo Urology and Nephrology Hospital, Ningbo, Zhejiang, China.

Competing interests None declared.

Patient consent for publication Not required.

Provenance and peer review Not commissioned; externally peer reviewed.

Data availability statement Data are available upon reasonable request. Data will be shared in the form of local and regional presentations and manuscript publications that will be publically available to all individuals in the scientific community. We will willingly share our knowledge, protocol, and expertise when asked. Requests for commercially based corporations will be negotiated by our institution's Technology Transfer Office.

Open access This is an open access article distributed in accordance with the Creative Commons Attribution Non Commercial (CC BY-NC 4.0) license, which permits others to distribute, remix, adapt, build upon this work non-commercially, and license their derivative works on different terms, provided the original work is properly cited, appropriate credit is given, any changes made indicated, and the use is non-commercial. See: http://creativecommons.org/licenses/by-nc/4.0/.

ORCID iD

Yufeng Huang http://orcid.org/0000-0001-9186-5269

\section{REFERENCES}

1 Molitch ME, DeFronzo RA, Franz MJ, et al. Nephropathy in diabetes. Diabetes Care 2004;27:S79-83.

2 Maahs DM, West NA, Lawrence JM, et al. Epidemiology of type 1 diabetes. Endocrinol Metab Clin North Am 2010;39:481-97. 
3 Osterby R, Bangstad HJ, Nyberg G, et al. On glomerular structural alterations in type-1 diabetes. Companions of early diabetic glomerulopathy. Virchows Arch 2001;438:129-35.

4 Østerby R, Hartmann A, Bangstad H-J. Structural changes in renal arterioles in type I diabetic patients. Diabetologia 2002;45:542-9.

5 Salmon AHJ, Satchell SC. Endothelial glycocalyx dysfunction in disease: albuminuria and increased microvascular permeability. $J$ Pathol 2012;226:562-74.

6 Augustin HG, Koh GY, Thurston G, et al. Control of vascular morphogenesis and homeostasis through the angiopoietin-Tie system. Nat Rev Mol Cell Biol 2009;10:165-77.

7 Maisonpierre PC, Suri C, Jones PF, et al. Angiopoietin-2, a natural antagonist for Tie2 that disrupts in vivo angiogenesis. Science 1997;277:55-60

8 Woolf AS, Gnudi L, Long DA. Roles of angiopoietins in kidney development and disease. J Am Soc Nephrol 2009;20:239-44.

9 Lim HS, Blann AD, Chong AY, et al. Plasma vascular endothelial growth factor, angiopoietin-1, and angiopoietin-2 in diabetes: implications for cardiovascular risk and effects of multifactorial intervention. Diabetes Care 2004;27:2918-24.

10 Yamamoto Y, Maeshima Y, Kitayama H, et al. Tumstatin peptide, an inhibitor of angiogenesis, prevents glomerular hypertrophy in the early stage of diabetic nephropathy. Diabetes 2004;53:1831-40.

11 Davis B, Dei Cas A, Long DA, et al. Podocyte-specific expression of angiopoietin-2 causes proteinuria and apoptosis of glomerular endothelia. J Am Soc Nephrol 2007;18:2320-9.

12 Jeansson M, Gawlik A, Anderson G, et al. Angiopoietin-1 is essentia in mouse vasculature during development and in response to injury. J Clin Invest 2011;121:2278-89.

13 Dessapt-Baradez C, Woolf AS, White KE, et al. Targeted glomerular angiopoietin-1 therapy for early diabetic kidney disease. J Am Soc Nephrol 2014;25:33-42.

14 Salmon AHJ, Neal CR, Sage LM, et al. Angiopoietin-1 alters microvascular permeability coefficients in vivo via modification of endothelial glycocalyx. Cardiovasc Res 2009;83:24-33.

15 Su D, Zhang N, He J, et al. Angiopoietin-1 production in islets improves islet engraftment and protects islets from cytokine-induced apoptosis. Diabetes 2007;56:2274-83.

16 Calderari S, Chougnet C, Clemessy M, et al. Angiopoietin 2 alters pancreatic vascularization in diabetic conditions. PLoS One 2012; 7:e29438.

17 Huang Y, Border WA, Yu L, et al. A PAl-1 mutant, PAl-1R, slows progression of diabetic nephropathy. J Am Soc Nephrol 2008;19:329-38.

18 Cho C-H, Kammerer RA, Lee HJ, et al. Comp-Ang1: a designed angiopoietin-1 variant with nonleaky angiogenic activity. Proc Natl Acad Sci U S A 2004;101:5547-52.

19 Cahoon JM, Rai RR, Carroll LS, et al. Intravitreal AAV2.COMP-Ang1 prevents neurovascular degeneration in a murine model of diabetic retinopathy. Diabetes 2015;64:4247-59.

$20 \mathrm{Yu}$ X, Seegar TCM, Dalton AC, et al. Structural basis for angiopoietin-1-mediated signaling initiation. Proc Natl Acad Sci U S A 2013;110:7205-10.

21 Lee S, Kim W, Moon S-O, et al. Renoprotective effect of COMPangiopoietin-1 in $\mathrm{db} / \mathrm{db}$ mice with type 2 diabetes. Nephrol Dial Transplant 2007;22:396-408.

22 Mori S, Wang L, Takeuchi T, et al. Two novel adeno-associated viruses from cynomolgus monkey: pseudotyping characterization of capsid protein. Virology 2004;330:375-83

23 Zhou G, Johansson U, Peng X-R, et al. An additive effect of eplerenone to ACE inhibitor on slowing the progression of diabetic nephropathy in the db/db mice. Am J Transl Res 2016;8:1339-54.

24 Zhou G, Liu X, Cheung AK, et al. Efficacy of aliskiren, compared with angiotensin II blockade, in slowing the progression of diabetic nephropathy in $\mathrm{db} / \mathrm{db}$ mice: should the combination therapy be a focus? Am J Transl Res 2015;7:825-40.

25 Zhou G, Cheung AK, Liu X, et al. Valsartan slows the progression of diabetic nephropathy in $\mathrm{db} / \mathrm{db}$ mice via a reduction in podocyte injury, and renal oxidative stress and inflammation. Clin Sci 2014;126:707-20.

$26 \mathrm{Gu} \mathrm{C}$, Zhang J, Noble NA, et al. An additive effect of anti-PAl-1 antibody to ACE inhibitor on slowing the progression of diabetic kidney disease. Am J Physiol Renal Physiol 2016;311:F852-63.

27 Huang Y, Noble NA. PAl-1 as a target in kidney disease. Curr Drug Targets 2007;8:1007-15.

28 Sanden SK, Wiggins JE, Goyal M, et al. Evaluation of a thick and thin section method for estimation of podocyte number, glomerular volume, and glomerular volume per podocyte in rat kidney with Wilms' tumor-1 protein used as a podocyte nuclear marker. J Am Soc Nephrol 2003;14:2484-93.

29 Guo J-K, Menke AL, Gubler M-C, et al. WT1 is a key regulator of podocyte function: reduced expression levels cause crescentic glomerulonephritis and mesangial sclerosis. Hum Mol Genet 2002:11:651-9.

30 Reiser J, von Gersdorff G, Loos M, et al. Induction of B7-1 in podocytes is associated with nephrotic syndrome. J Clin Invest 2004;113:1390-7.

31 Zanone MM, Favaro E, Camussi G. From endothelial to beta cells: insights into pancreatic islet microendothelium. Curr Diabetes Rev 2008;4:1-9

32 Narayanan S, Loganathan G, Dhanasekaran M, et al. Intra-islet endothelial cell and $\beta$-cell crosstalk: implication for islet cell transplantation. World J Transplant 2017;7:117-28.

33 Brissova M, Shostak A, Fligner CL, et al. Human islets have fewer blood vessels than mouse islets and the density of islet vascular structures is increased in type 2 diabetes. $J$ Histochem Cytochem 2015:63:637-45.

34 Hogan MF, Hull RL. The islet endothelial cell: a novel contributor to beta cell secretory dysfunction in diabetes. Diabetologia 2017;60:952-9

35 Kawasaki F, Matsuda M, Kanda Y, et al. Structural and functional analysis of pancreatic islets preserved by pioglitazone in $\mathrm{db} / \mathrm{db}$ mice. Am J Physiol Endocrinol Metab 2005;288:E510-8.

$36 \mathrm{Li} \mathrm{X}$, Zhang L, Meshinchi S, et al. Islet microvasculature in islet hyperplasia and failure in a model of type 2 diabetes. Diabetes 2006;55:2965-73.

37 Brissova M, Shostak A, Shiota M, et al. Pancreatic islet production of vascular endothelial growth factor-a is essential for islet vascularization, revascularization, and function. Diabetes 2006;55:2974-85.

38 Cai Q, Brissova M, Reinert RB, et al. Enhanced expression of VEGF-A in $\beta$ cells increases endothelial cell number but impairs islet morphogenesis and $\beta$ cell proliferation. Dev Biol 2012;367:40-54.

39 Shah P, Lueschen N, Ardestani A, et al. Angiopoetin-2 signals do not mediate the Hypervascularization of islets in type 2 diabetes. PLoS One 2016;11:e0161834.

40 Park HS, Kim HZ, Park JS, et al. $\beta$-Cell-Derived Angiopoietin-1 Regulates Insulin Secretion and Glucose Homeostasis by Stabilizing the Islet Microenvironment. Diabetes 2019;68:774-86.

41 Rasul S, Reiter MH, Ilhan A, et al. Circulating angiopoietin-2 and soluble Tie-2 in type 2 diabetes mellitus: a cross-sectional study. Cardiovasc Diabetol 2011;10:55

42 Jung YJ, Park W, Nguyen-Thanh T, et al. COMP-angiopoietin-1 mitigates changes in lipid droplet size, macrophage infiltration of adipose tissue and renal inflammation in streptozotocin-induced diabetic mice. Oncotarget 2017;8:94805-18. 